$\mathbb{T}$ periodica polytechnica

\author{
Electrical Engineering \\ and Computer Science \\ 56/4 (2012) 105,111 \\ doi: 10.3311/PPee.7164 \\ http://periodicapolytechnica.org/ee \\ Creative Commons Attribution (1) \\ RESEARCH ARTICLE \\ Viktor Kálmán / László Vajta \\ Received 2012-09-28
}

\section{Designing and tuning a brake assistant for omnidirectional wheels}

\begin{abstract}
Although the invention of the special wheels that move omnidirectional vehicles dates back to the seventies, advances in mechatronics, and control technology keep them constantly on the drawing table of engineers working with mobile robots. The need for eliminating human error becomes ever so obvious when human operators are managing expensive and/or powerful machinery, as the cost of failure can be very high. Omnidirectional platforms are not immune to human error either, however due to their unique working principle they require customized methods. This article presents a trajectory controller for omnidirectional transport robots that is able to correct their trajectory during braking even when high disturbances are present. A method for tuning the controller to achieve a desired behavior is presented. The results are demonstrated by simulation, in Modelica - Dymola environment.
\end{abstract}

\section{Keywords}

omnidirectional wheels $\cdot$ brake assistant $\cdot$ sliding mode control $\cdot$ Modelica simulation

\section{Viktor Kálmán}

Department of Control Engineering and Information Technology, Faculty of Electrical Engineering and Informatics, Budapest University of Technology and Economics, Magyar Tudósok körútja 2., H-1117 Budapest, Hungary e-mail: kalman@iit.bme.hu

\section{László Vajta}

Department of Control Engineering and Information Technology, Faculty of Electrical Engineering and Informatic, Budapest University of Technology and Economics, Magyar Tudósok körútja 2., H-1117 Budapest, Hungary

\section{Introduction}

When human operators are managing expensive and/or powerful machinery, the importance of preventing failure is paramount. Complex machines usually require complex control interfaces to exploit their capabilities to the full extent, however complexity may do more harm than good, that is why it is best to hide the complexity behind a clean and simple user interface. A good example of this are transparent corrective systems such as ESP or ABS that we meet every day. ESP for instance simultaneously controls the brakes of four wheels, it is easy to see why a driver would be unable to achieve similar control, especially at speed. These systems increasingly find their way into robotic applications. Omnidirectional platforms are not immune to human error either, however due to their different working principle they require different methods. This article describes a controller that is designed to help avoid swerving of omnidirectional platforms during braking, due to uneven load distribution, or disturbances in the force.

\subsection{Problem description}

Due to advances in control theory, mechatronics and manufacturing technology omnidirectional wheels - invented in the seventies [5] - are living their renaissance. They have a rich history in the literature, they have been used for various tasks, and many different embodiments are known [3, 10]. Their use ranges from robotic soccer applications, through industrial heavy load transporters [11], and vehicle simulators [1], to leisure and educational projects [2, 9].

The wheels themselves consist of several - usually rubber coated - free rolling rollers (Fig. 1), the most popular roller angles are $45^{\circ}$ and $0^{\circ}$, the former is usually called Mecanum, or Swedish wheel, the latter is usually called Omni-wheel. Obviously other angles are possible, but their usage leads to unjustifiably more complex mechanical design and control. Also, angles close to $90^{\circ}$ are totally useless [6]. The rollers are spinning freely, only the main axis is powered.

Due to their design omnidirectional wheels in general have a one dimensional force generation capability, they can only exert substantial force parallel to the roller axes, this is the very 


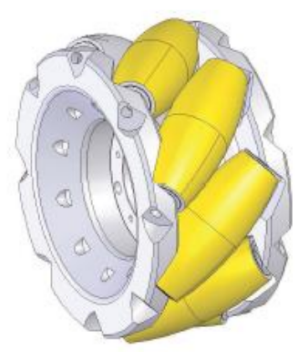

Fig. 1. Cad drawing of a mecanum wheel [14]

attribute that allows omnidirectional movement. As a consequence such platforms can be pushed in any direction when their wheels are rolling free. The main problem is that they tend not to keep their orientation during braking. This is caused by slight differences between wheel forces arising from uneven load distribution, or ground friction variations. These unbalanced forces create a resulting torque, and thus an angular acceleration around the center of gravity during braking.

\section{Brake assist controller}

To handle the problem described above a solution is proposed in this section. First the underlying modeling concept is introduced then a sliding mode controller is developed. The second part of this section deals with the possibilities of tuning the controller, to best fit user preferences.

\subsection{Platform model}

To approach this control problem let's consider the forces arising during braking. This is illustrated on Fig. 2 on two wheels of a general omnidirectional platform. It is assumed that the platform moves on flat ground, thus having three degrees of freedom.

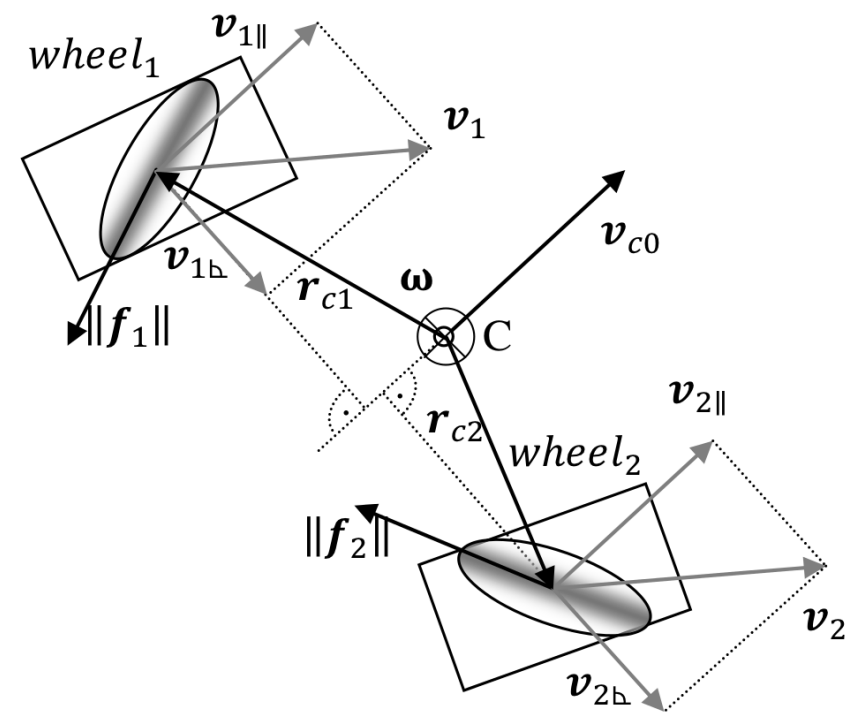

Fig. 2. Velocity and force vectors of a braking omnidirectional platform

The velocity vectors at the wheel centers can be described in terms of platform linear and angular velocity:

$$
\mathbf{v}_{i}=\mathbf{v}_{C}+\omega \times \mathbf{r}_{c i}
$$

where $\mathbf{v}_{i}$ are velocities of wheel centers, $\mathbf{v}_{C}$ is platform velocity, $\omega$ is platform angular velocity; $\mathbf{r}_{c i}$ are vectors from the platform geometrical center to wheel centers. Force vectors always arise to oppose instantaneous wheel velocities, thus a unit vector pointing in the direction of the $\mathbf{f}_{i}$ friction forces can be calculated as follows:

$$
\left\|\mathbf{f}_{i}\right\|=-\operatorname{sign}\left(\mathbf{v}_{i} \mathbf{n}_{w i}\right) \mathbf{n}_{w i}
$$

where $\mathbf{n}_{w i}$ are unit vectors pointing in the direction of the roller axes on the ground. The magnitudes of these forces depend on the load of the particular wheel, the material and the ground. The dynamics of the platform can be described by Newton's law

$$
\begin{gathered}
m \dot{\mathbf{v}}_{C}=\sum_{i} \mathbf{f}_{i} \\
\mathbf{J}_{c} \dot{\omega}=\sum_{i} \mathbf{f}_{i} \times \mathbf{r}_{C i}
\end{gathered}
$$

In these dynamic equations $m$ stands for mass and $\mathbf{J}_{c}$ stands for the inertia matrix of the platform. Both of them are uncertain and in case of a transport robot they change each time the payload is changed. To be able to control this uncertain MIMO system I decided to use sliding mode control, as suggested by many in the literature [7, 12, 15, 16].

\subsection{Sliding mode controller}

The idea behind sliding mode control is to constrain the plant to a prescribed trajectory - the sliding manifold - by choosing the control gains, to drive the state variables towards it. This is achieved by discontinuous changes in the gains [7].

In the case of a braking omnidirectional vehicle the control goal is twofold,

- Goal 1: it is important to avoid directional change in the platforms movement

- Goal 2: obviously it is also important to try and stop the platform as soon as possible

The goals are simple but several problems pose difficulties in reaching them:

- Since the wheels cannot be turned, the direction of force they generate only depends on the velocity direction of the contact patch, therefore the only option to assert control is to modulate the braking force.

- Force generation is passive in a sense that it can only act to decrease velocity, and not to increase it.

- Generally the friction forces of the wheels are not parallel with wheel velocities, therefore they will act to divert the platform from its original path. 
- From equations (1), (3), 4) it is clear that all $\mathbf{f}_{i}$ effects all $\mathbf{v}_{\mathbf{i}}, \mathbf{v}_{\mathbf{c}}$ and $\omega$ consequently it is generally not possible to affect a single degree of freedom of the platform independently.

These considerations mean that brake modulation has to depend on the direction of the friction force, if it drives the platform towards a given trajectory then it needs to be applied, otherwise, the wheel needs to be rolling free. To construct the sliding surface we have to consider the nature of trajectory we would like to track. Since the magnitudes of the forces are uncertain the trajectory cannot be defined as an exact function of time. At the moment when braking starts the platform has $\mathbf{v}_{0}$, $\omega_{\mathbf{0}}$ initial velocities, the platform has to gradually lose its angular velocity and it cannot gain velocity in the direction perpendicular to $\mathbf{v}_{0}$ i.e. it cannot divert from the trajectory before braking.

To translate these considerations into control rules the sliding surface can be written in the following form for each wheel:

$$
s_{i t o t}=s_{i \|}+s_{i \perp}+s_{i \omega}
$$

where the three components correspond to wheel velocity components parallel and perpendicular to $\mathbf{v}_{0}$ and a component due to $\omega$. Each component signifies whether the given $-i$-th - wheel is able to drive that velocity component to zero, therefore the control law can be constructed to apply the $i$-th brake when its corresponding $s$ function is negative:

$$
\mathbf{u}_{i}=\mathbf{f}_{i}\left(\zeta\left(s_{\text {itot }}\right)\right)
$$

where

$$
\zeta(x)=\left\{\begin{array}{ll}
0 & \text { for } \forall x \geq 0 \\
1 & \text { for } \forall x<0
\end{array} x \in \mathcal{R}\right.
$$

Components of $s_{i t o t}$ can be calculated the following way:

$$
s_{i \perp}=\left\|\mathbf{f}_{i}\right\| \mathbf{v}_{i \perp}
$$

where $\mathbf{v}_{i \perp}$ is the projection of $\mathbf{v}_{i}$ on $\mathbf{n}_{w, i}$ the direction of the roller axis of the wheel. Similarly

$$
s_{i \omega}=\left\|\beta_{w i}\right\| \omega\left|r_{c i}\right|
$$

where $\left|r_{c i}\right|$ is the distance from the platform center to the wheel center, and

$$
\left\|\beta_{w i}\right\|=\mid \mathbf{f}_{i}\|\times\| \mathbf{c i} \|
$$

is the direction of the torque generated by $\mathbf{f}_{i}$.

$s_{i \|}$ is a different matter, the previously discussed two components change from wheel to wheel, the parallel component however can be decreased by any wheel in most cases, so if we activate the brake according to the sign of $s_{i \|}$ it will always be on, no modulation will take place, same as the uncontrolled case. Its general form can be written as follows:

$$
s_{i \|}=\psi\left(\left\|\mathbf{f}_{i}\right\|, \mathbf{v}_{i \|}\right)\left\|\mathbf{f}_{i}\right\| \mathbf{v}_{i \|}
$$

where $\mathbf{v}_{i \|}$ is the projection of $\mathbf{v}_{i}$ on $\mathbf{u}_{w i}$ the direction perpendicular to the roller axis of the wheel, $\psi()$ is a weighing function. Different approaches can be taken to deal with this component, these are discussed in section 2.3 , with the different effects demonstrated trough examples.

\subsubsection{Feedback}

The state variables $\mathbf{v}_{c}$ and $\omega$ are assumed to be measurable, by any means convenient, however we suggest a method similar to the principle used in optical mice, described in [13]. The advantage of the method is that velocity measurement is independent from wheel rotation.

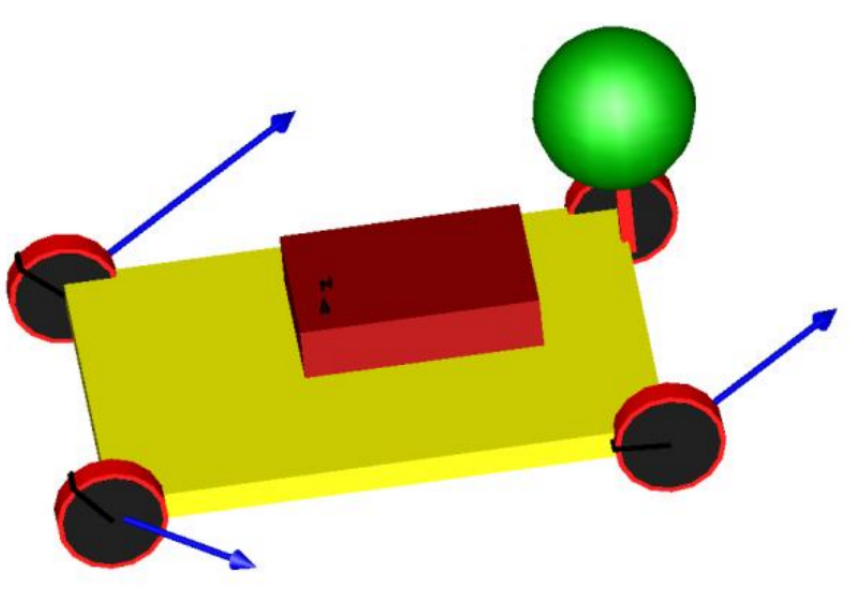

Fig. 3. Mecanum platform in simulation

\subsubsection{Stability}

To find out if the closed loop control system is stable, the so called passivity approach [7] (p. 436) can be used. It means that if the components in the feedback connection are passive, in the sense that they do not generate energy on their own, then it is intuitively clear that the system will be passive. Generally a system is called passive if there exists a storage function $V(t) \geq$ 0 , such that for all $t_{0}<t_{1}$,

$$
V\left(t_{1}\right) \leq V\left(t_{0}\right)+\int_{t_{0}}^{t_{1}} y(t) u(t) d t
$$

where $y$ is the system output and $u$ is the input respectively. This equation simply states, that the energy $(V(t))$ of the system consists of the initial energy plus the supply rate $y u$. If the equality holds, the system is lossless, if it is a strict inequality then the system is dissipative.

If we set the feedback to $u=-K y$, where $K$ is a positive gain then it is guaranteed that the system energy remains bounded, thus the feedback system is stable. Considering an omnidirectional platform in the context of the brake assist system, energy is stored in the form of kinetic energy. This can be written in the following form:

$$
V=\frac{M v_{c}^{2}}{2}+\frac{J \Omega^{2}}{2}
$$


Fig. 4. Snapshots from a braking maneuver

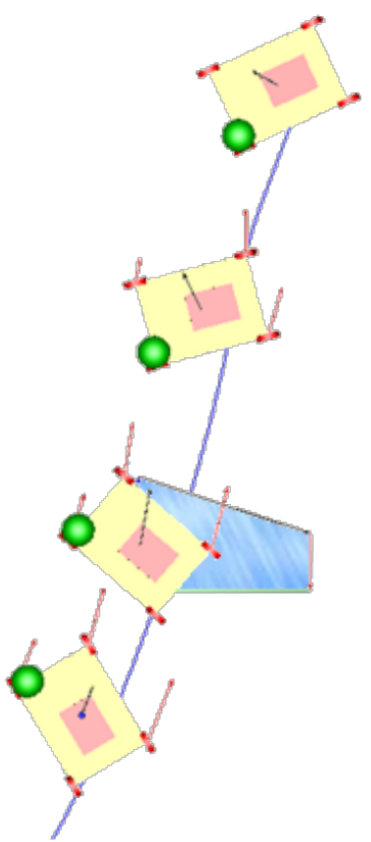

(a) no assistant

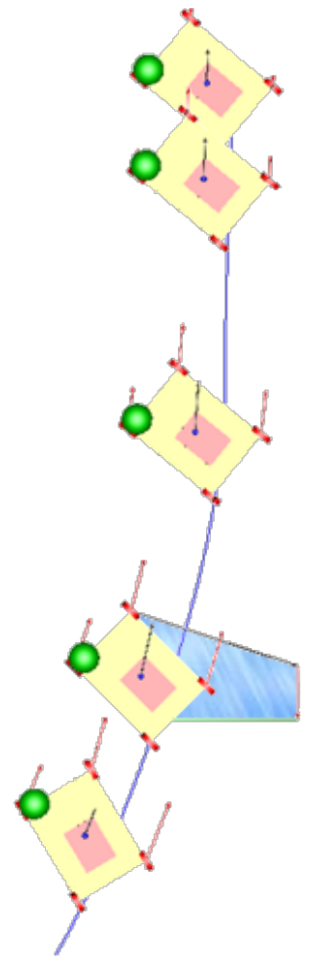

(b) 0 weighing function

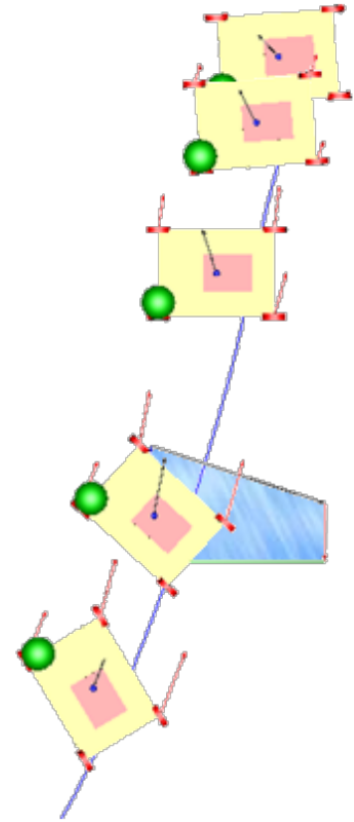

(c) cosine weighing function considering the linear and rotational kinetic energies. The "supply rate" - in this case would be more appropriately called the "dissipation rate" - is the product of friction force and velocity which is essentially the power dissipated by braking the wheels. The goal of the braking maneuver is to drive the kinetic energy to zero. Brake forces are always generated to oppose movement, causing negative acceleration. Since no energy is put in the system by the brake forces, it is evident that the system is passive and the inequality (8) holds true for $\forall t_{0}, t_{1}$ time instants. However for a braking maneuver a strict inequality is a necessary condition for the vehicle to stop in a reasonable distance, which means that if we neglect small frictional effects such as rolling resistance and bearing friction etc. a strict inequality can only be achieved if it is guaranteed that:

- at least one brake is actuated at any time instant

- with its active direction at an angle other than $90^{\circ}$ to the direction of movement

Part of the second criterion can be guaranteed by design, if the vehicle is created so that it is controllable [6]. The first criterion means that the error function of at least one wheel has to be negative at all times. This is easily guaranteed if we add a rule for the controller that turns every brake on if $\forall s_{\text {itot }} \geq 0$.

\subsection{Brake effect tuning}

The sliding surface in equation (5) has three components. The role of $s_{i \perp}$ and $s_{i \omega}$, is easy to see, they are responsible for eliminating rotation and translation of the platform, $s_{i \|}$ however is somewhat different. Let's investigate the effect of $s_{i \|}$ on the braking maneuver in more detail, with the help of simulation.

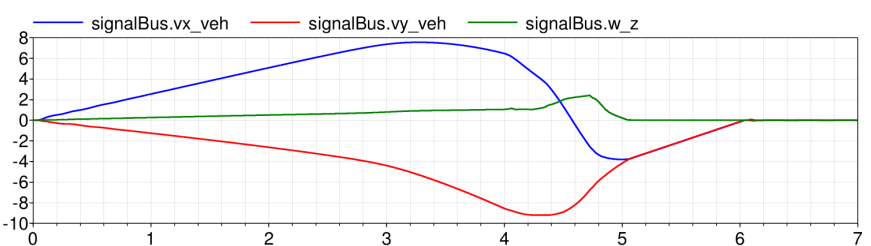

Fig. 5. Linear and angular velocities of the unassisted vehicle

\subsubsection{Simulation}

I created a Mecanum wheeled forklift model, and executed experiments to demonstrate the capabilities of the controller. The platform has four wheels, the vehicle body is represented by a point mass with inertia, and the load is represented by a simple point mass. Both the position and value of the masses, as well as vehicle dimensions are configurable. The platform is shown on Fig. 3 . The body is represented by the red brick and the load is the green sphere. It is easy to see, that the center of gravity is offset to the front left of the platform, simulating a carelessly loaded heavy cargo. The wheels of the platform are based on the article [8] using a modified TMEasy model [4] for dynamic force generation.

The floor is flat and uniform, and patches of different friction coefficient can be defined. It can be thought of as a warehouse floor, for example with some spilled oil. The platform is slowed by four disc brakes, with cutoff valves. The main body has a mass of $600 \mathrm{~kg}$ and the load is $200 \mathrm{~kg}$, so a heavily loaded small forklift is considered.

The experiment consists of a constant acceleration part, where the vehicle is accelerated in $\mathrm{x}, \mathrm{y}$ directions in local coordinates, 
Fig. 6. Velocities and error functions for the assisted maneuver -0 weighing function
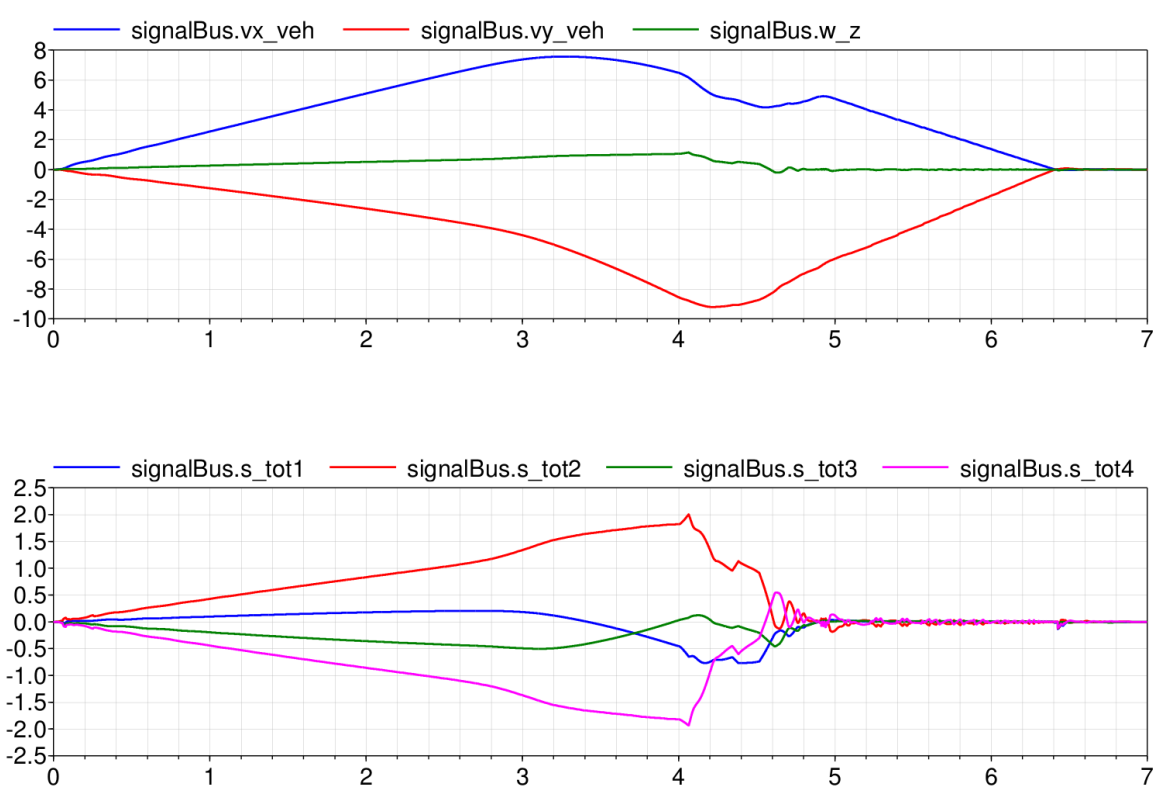

Fig. 7. Velocities and error functions for the assisted maneuver - cosine weighing function
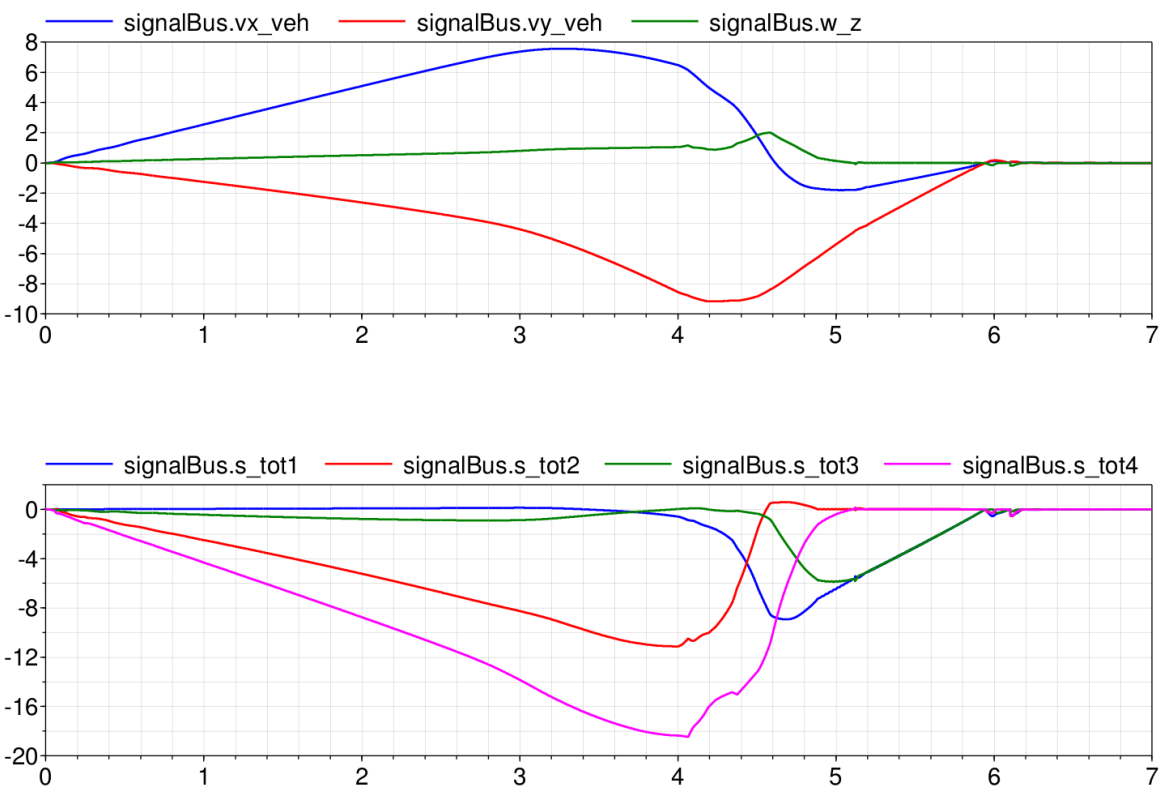

with constant angular acceleration. At a given point the brakes are actuated, simulating an emergency situation. To introduce a disturbance, a patch of smaller friction coefficient is placed in the way of the braking vehicle, so that it falls under the rear wheels effectively increasing the swerving effect. The simulated run can be seen on Fig 4 (a).

State variables $\mathbf{v}_{c}, \omega$ of the platform can be seen on Fig. 5 The braking starts at $4 \mathrm{~s}$ and brake force is linearly increased until the maximum at $5 \mathrm{~s}$. The effect of the "oil spill" is clearly visible on the angular velocity ( $w_{-} z$ in the figure) from $4.3 \mathrm{~s}$ when the vehicle crosses it.

\subsubsection{No parallel component}

Since the model of a general omnidirectional platform is input - output cross coupled as it can be seen from the dynamic model and the general kinematic model [6], slowing one wheel will usually affect both the parallel and perpendicular velocity component, so when the perpendicular and the angular velocity components are driven to zero, the parallel component will vanish as well. Degenerate scenarios are possible, for instance the case of a Mecanum platform when it stops from a straight $45^{\circ}$ movement, no perpendicular nor any angular velocity components are present, all $s_{i t o t} \geq 0$. For this case a rule can be implemented that actuates all brakes, guaranteeing the platform to stop in a finite time. Snapshots from the experiment can be seen on Fig. 4(b) velocities and error functions can be seen on Fig. 6. The control rule "punishes" angular velocity and it can be seen on the graph of the state variables that it effectively transfers rotational energy into linear kinetic energy, which causes the platform to stop in a longer distance. 
Tab. 1. Comparison of controller implementations

\begin{tabular}{lccccc}
\hline & unassisted & $\phi=0$ & $\begin{array}{c}\phi=\cos (\gamma) \\
k=1\end{array}$ & $\begin{array}{c}\text { enhanced } \phi \\
\mathrm{k}=7\end{array}$ & enhanced $\phi$ \\
\hline orientation change & $83.8^{\circ}$ & $18.9^{\circ}$ & $63.2^{\circ}$ & $52^{\circ}$ & $26.3^{\circ}$ \\
\hline stopping distance & $10 \mathrm{~m}$ & $12.2 \mathrm{~m}$ & $9.7 \mathrm{~m}$ & $9.3 \mathrm{~m}$ & $10.4 \mathrm{~m}$ \\
\hline
\end{tabular}

\subsubsection{Parallel component as a function of velocity direction}

As it can be seen from the previous experiment (Fig.4(b)) orientation of the platform was kept at the cost of increased stopping distance. For most situations it is obviously unacceptable. To correct the behavior, an error component corresponding to the parallel velocity component needs to be added. It needs to be angle dependent, so that Goal 2 only dominates the error function when the given wheel is most effective i.e. its active direction is close to parallel with its velocity $\left(\mathbf{n}_{w i} \| \mathbf{v}_{i}\right)$.

A cosine function of the angle between the active direction of the wheel and the velocity of its center seems to be a good candidate, as it yields 1 when the vectors are parallel and 0 when they are perpendicular. The maneuver can be seen on Fig. 4(c) and the velocity and error functions on Fig. 7 7 The behavior of the vehicle and the velocity curves are very similar to the unassisted case, swerving however is clearly reduced.

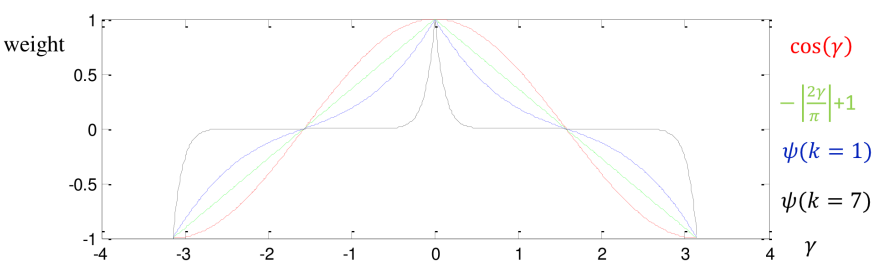

Fig. 8. Enhanced weighing functions for the parallel component

\subsubsection{Enhanced directional dependence}

The situation can be further enhanced by tuning the weighing function of the parallel component, as cosine decays too slow around zero letting $s_{\|}$dominate over a wide angle region. The next idea is to modify the weighing function to something that decays faster letting Goal 1 dominate when $\left(\mathbf{n}_{w i} \nVdash \mathbf{v}_{i}\right)$. The simplest idea is to mirror the cosine function on the line connecting its maxima with its minima. This is achieved by the following function:

$$
\psi=\left(-\left|\frac{2 \gamma}{\pi}\right|+1+\left(-\left|\frac{2 \gamma}{\pi}\right|+1-\cos (\gamma)\right)\right)^{k}, \gamma \in[-\pi, p i]
$$

or after simplification:

$$
\psi=\left(-2\left|\frac{2 \gamma}{\pi}\right|+2-\cos (\gamma)\right)^{k}
$$

where $k$ is a tuning constant, an odd positive integer. The function is illustrated on Fig. 8 with $k=1$ and 7. (The range we are interested in is marked in equation $(10)$, the graph is wider for better visibility.)

The experiment with the modified weighing function can be seen in Fig. 9(b) and 9(c). For comparison Fig. 9(a) shows the experiment with the cosine weighing function (same as Fig. 4(c)), it can be seen that with $\mathrm{k}=1$ better directional control is maintained and the vehicle stops in a short distance while with $\mathrm{k}=7$ we are getting closer to 0 weighing function case, where the orientation is the closest to that at the start of the maneuver, but the stopping distance is the longest. It is clear that with the choice of $k$ the controller can be tuned to the users needs.

Velocities and error function for the case of $\mathrm{k}=1$ can be seen on Fig. 10 The beneficial effects on the state variables are clearly visible, compared to Fig. 6 and Fig. 77 The 0 weighing function drives the angular velocity to zero effectively, but it does not affect the linear velocity much. The cosine weigh function allows too much angular velocity, while the enhanced controller affects both linear and angular velocities.
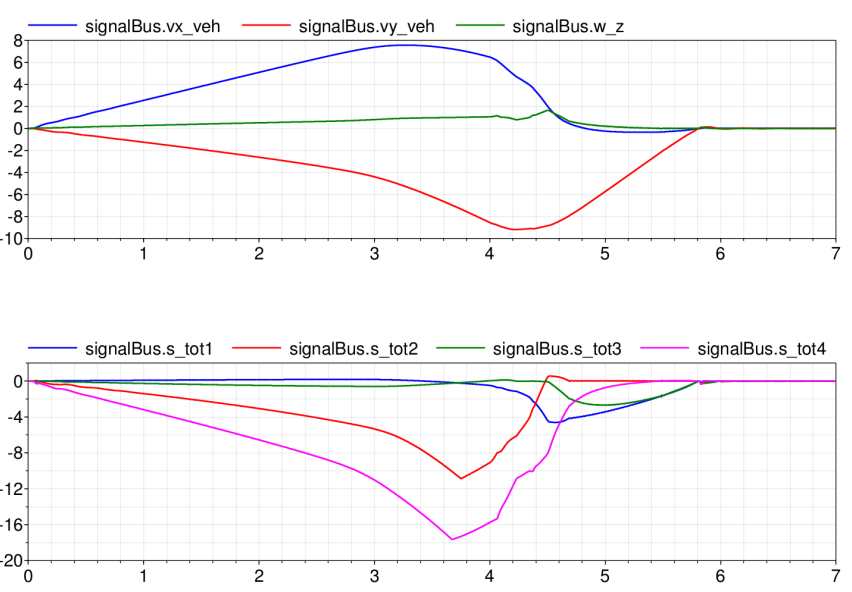

Fig. 10. Velocities and error functions for the assisted maneuver - enhanced weighing function $k=1$

\section{Conclusion}

A braking omnidirectional transport robot can be approached as an uncertain nonlinear MIMO control problem. I created a sliding mode controller that effectively keeps the orientation of the platform during braking, by eliminating velocity components of the wheels that are not in the direction of platform velocity. The resulting controller is able to maintain the orientation of the vehicle during braking, and stops the vehicle thanks to the cross coupled nature of the general omnidirectional vehicle. The goal is reached at the expense of increased braking distance, compared to the unassisted case. I proposed a method to incorporate a component into the control rule that represents a healthy compromise, by maintaining directional control, while keeping brake distance at a value similar to the unassisted case. Table 1 shows the summary of the results with this particular robot, for comparison. Both orientation change and stopping 
Fig. 9. Comparison of weighing functions

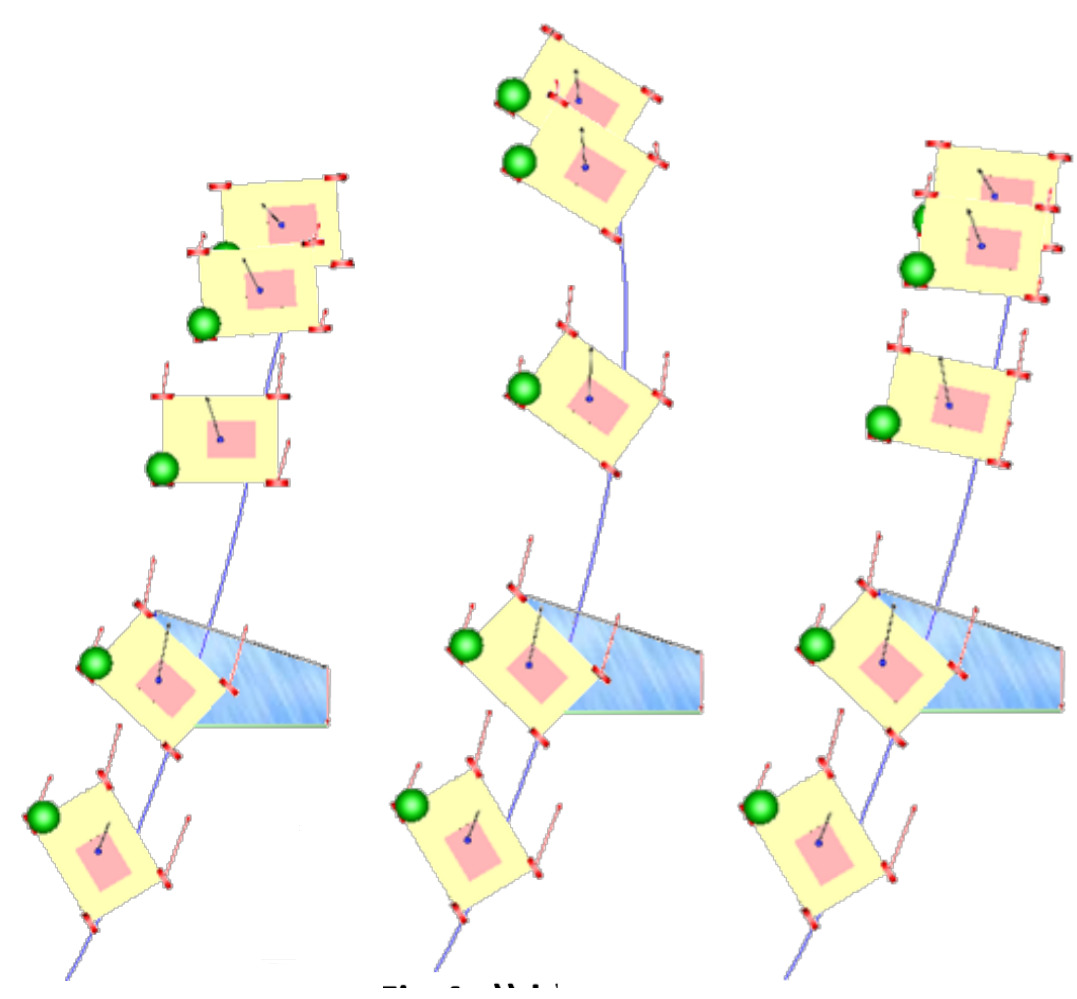

(a) cosine (b) enhanced function $k=1$ (c) enhanced function $k=7$ distance are calculated between the start of braking and the final position.

The proposed weighing function depends on the angle between roller direction and instantaneous velocity of a wheel center, and it can be tuned by changing the value of to favor directional control or stopping distance. The controller shows great disturbance rejection, due to the fact that it operates independent from the vehicle's dynamic model, it only uses kinematic information of the platform.

\section{References}

1 Ahmad R, Toonders P, Hayes MJD, Langlois RG, Atlas mecanum wheel jacobian empirical validation, CSME International Congress, (2012).

2 Bjärenstam MJ, Lennartsson M, Development of a ball balancing robot with omni wheels, 2012. Master's thesis.

3 Brunhorn J, Tenchio O, Rojas R, A Novel Omnidirectional Wheel Based on Reuleaux-Triangles, In: Robocup 2006: Robot soccer world cup, SpringerVerlag; Berlin, Heidelberg, 2007, pp. 516-522, DOI 10.1007/978-3-54074024-7_53.

4 Hirschberg W, Rill G, Weinfurter H, Tire model TMeasy, VEHICLE SYSTEM DYNAMICS, 45, (2007), 101-119, DOI $10.1080 / 00423110701776284$.

5 Ilon BE, Wheels for a course stable selfpropelling vehicle movable any desired direction on the ground or some other base, (1975). US Patent No. 3876255 .

6 Indiveri G, Swedish wheeled omnidirectional mobile robots: Kinematics analysis and control, IEEE Transactions on Robotics, 25, (2009), 164-171, DOI 10.1109/TRO.2008.2010360.

7 Khalil HK, Nonlinear systems, 2nd edn., Prentice-Hall, 2002, ISBN 0-13228024-8.

8 Kálmán V, Juhász T, Vajta L, Schmucker U, Mecanum wheel library in modelica, 15. IFF-Wissenschaftstage: Digitales Engineering zum planen, testen und betreiben technischer systeme, (2012).
9 Kumaga M, Ochiai T, Development of a robot balanced on a ball - application of passive motion to transport, IEEE International Conference on Robotics and Automation, 2009. ICRA '09, ( may 2009), 4106-4111, DOI 10.1109/ROBOT.2009.5152324.

10 Pin FG, Killough SM, A new family of omnidirectional and holonomic wheeled platforms for mobile robots, IEEE Transactions on Robotics and Automation, 10(4), (aug 1994), 480-489, DOI 10.1109/70.313098.

11 Ruf D, Tobolár J, Omnidirektionale fahrzeuge für schwerlasttransport in produktion und logistik, Logistik und Verkehr in Bayern, 12, (2011), 34-35.

12 Sandabanovic A, Variable structure systems with sliding modes in motion control - a survey, IEEE Transactions on Industrial Informatics, 7(2), (may 2011), 212-223, DOI 10.1109/TII.2011.2123907.

13 Takács T, Kálmán V, Vajta L, Frontiers in Robotics, Automation and Control, Optical Speed Measurement and applications, (2008), 165-188, DOI $10.5772 / 6327$.

14 Tobolár J, Herrmann F, Bünte T, Object-oriented modelling and control of vehicles with omni-directional wheels, Computational mechanics 25th conference with international participation, (November 9-11 2009).

15 Vecchio C, Sliding Mode Control: theoretical developments and applications to uncertain mechanical systems, $\mathrm{PhD}$ thesis, 2008.

16 Young KD, Utkin VI, Ozguner U, A control engineer's guide to sliding mode control, IEEE Transactions on Control Systems Technology, 7(3), (may 1999), 328-342, DOI 10.1109/87.761053. 